\title{
O uso de ambiente de geometria dinâmica como subsídio para a caracterização das funções quadráticas
}

\author{
Alan de Oliveira $\quad$ Fabio Dorini
}

\begin{abstract}
Resumo
No processo de ensino e aprendizagem do conceito de função quadrática abordado no ensino médio, tem-se constatado que o tratamento apenas manipulativo da expressão algébrica associada à função pode limitar o desenvolvimento do raciocínio funcional no que diz respeito à sua concepção conceitual. Destarte, o uso de ambiente de geometria dinâmica para a construção desse conhecimento propicia aos alunos a possibilidade de superar essa limitação, permitindo-lhes refletir como a variação de uma grandeza depende da variação de outra. Este artigo, oriundo de parte de uma Dissertação de Mestrado [3] do PROFMAT ${ }^{1}$, sugere atividades desenvolvidas em GeoGebra que, baseadas no teorema de caracterização das funções quadráticas, abrangem a identificação de funções quadráticas em situações que relacionam objetos geométricos sem o uso, a priori, das representações algébricas e/ou gráficas.
\end{abstract}

Palavras-chave: ensino de Matemática; função quadrática; GeoGebra.

\section{Abstract}

In the process of teaching and learning the concept of quadratic function addressed in high education, it has been found that the manipulative treatment of algebraic expression associated with function may limit the development of functional reasoning with respect to its conceptual conception. Thus, the use of a dynamic geometry environment for the construction of this knowledge allows students to overcome this limitation, allowing them to reflect how the variation of one quantity depends on the variation of another. This article, from a Master's Dissertation [3] of PROFMAT, suggests activities developed in GeoGebra that, based on the theorem of characterization of quadratic functions, cover the identification of quadratic functions in situations that relate geometric objects without the use, the priori, of algebraic and/or graphic representations.

Keywords: Mathematics teaching; quadratic function; GeoGebra.

\section{Introdução}

\footnotetext{
${ }^{1}$ Mestrado Profissional em Matemática em Rede Nacional - <http://www.profmat-sbm.org.br/>.
} 
O ensino de funções no ensino médio, praticado sob a perspectiva apenas da formalização matemática, pode-se constituir em obstáculo de difícil transposição no que diz respeito à compreensão de seu conceito. A abordagem de forma mecanizada com pouca reflexão na qual se desenvolve o domínio de técnicas, fórmulas e procedimentos de manipulações simbólicas e numéricas pode levar os alunos a associarem ao conceito apenas a ideia de correspondência. Desta forma, inibe-se o desenvolvimento de uma disposição e forma de pensar em que possam, constantemente, buscar e examinar diferentes tipos de relações, conjecturar, utilizar diferentes sistemas de representação, estabelecer conexões e empregar vários argumentos.

Os ambientes de geometria dinâmica permitem a abordagem do conceito de função em situações nas quais os alunos desenvolvem a percepção intuitiva da ideia de variação. Nesses ambientes, pode-se explorar o conceito investigando-se a relação de dependência entre objetos geométricos (basicamente, comprimentos e áreas). Nessas relações a dependência entre objetos ocorre naturalmente. Por exemplo, se construirmos um retângulo cujo comprimento da altura é fixado, pode-se observar as variações da área em função do comprimento da base do retângulo [1]. Ou, se construirmos um quadrado inscrito em um círculo, o lado e a área do quadrado são funções do raio do círculo. Assim, através do estudo dessas variações, é possível tentar inferir o tipo de função que relaciona essas grandezas geométricas, sem deduzir a expressão algébrica ou construir o gráfico associados.

O GeoGebra ${ }^{2}$ é um aplicativo livre de geometria dinâmica, criado em 2001 por Markus Hohenwarter, desenvolvido para o ensino e aprendizagem da Matemática nos vários níveis escolares. Tal aplicativo associa ferramentas algébricas e geométricas possibilitando, em um mesmo ambiente, construir figuras em geometria dinâmica que representam, segundo [1], ,

\begin{abstract}
"uma classe de objetos geométricos definida por propriedades e relações comuns que se preservam quando arrastados na tela, permitindo ao usuário investigar um grande número de exemplos e explorar conjecturas, constituindo uma preparação para o exercício de argumentação matemática".
\end{abstract}

A principal característica do GeoGebra, segundo o seu idealizador, consiste na percepção dupla dos objetos: cada expressão na janela de álgebra corresponde a um objeto na janela de visualização gráfica e vice-versa. Por um lado, o GeoGebra possui todas as ferramentas tradicionais de um aplicativo de geometria dinâmica: pontos, segmentos, retas, circunferências, etc. Por outro, equações e coordenadas podem ser inseridas diretamente. Assim, o GeoGebra tem a vantagem didática de apresentar, ao mesmo tempo, duas representações diferentes de um mesmo objeto que interagem entre si: sua representação geométrica e sua representação algébrica, oferecendo, portanto, diversas possibilidades para a exploração pedagógica.

O uso do GeoGebra, cujos recursos disponibilizados facilitam a exploração algébrica, gráfica e a utilização de uma planilha de cálculos, de forma simultânea, permite estabelecer uma abordagem pouco, ou quase nada, explorada do conceito de função quadrática em situações nas quais se caracterizam uma relação de dependência funcional entre objetos geométricos.

Em vista disso, este artigo apresenta atividades desenvolvidas em GeoGebra cujo objetivo é auxiliar o professor de Matemática do ensino médio no tratamento do conceito de função quadrática. Baseado no teorema de caracterização das funções quadráticas, propõe a construção do conheci-

\footnotetext{
${ }^{2}<$ http://www.geogebra.org/>.
} 
mento através de atividades que visam explorar ideias intuitivas de variação e dependência entre objetos geométricos sem a mediação, a priori, de representações algébricas e/ou gráficas.

O teorema a seguir apresenta condições para que um problema possa ser modelado por uma função quadrática. O enunciado foi estabelecido com base em [2]. As atividades que serão propostas suscitam conjecturas cuja validade, ou não, é fundamentada nas condições explicitadas no referido teorema.

\section{Teorema de caracterização das funções quadráticas}

Teorema 1. Uma função contínua $f: \mathbb{R} \rightarrow \mathbb{R}$ é quadrática se, e somente se, toda progressão aritmética não constante $x_{1}, x_{2}, \ldots, x_{n}, \ldots$ é transformada por $f$ numa progressão aritmética de segunda ordem não degenerada $y_{1}, y_{2}, \ldots, y_{n}, \ldots$, em que $y_{1}=f\left(x_{1}\right), y_{2}=f\left(x_{2}\right), \ldots, y_{n}=f\left(x_{n}\right), \ldots$.

Para a compreensão desse teorema pressupõe-se desenvolvida a habilidade de conversão entre diferentes linguagens. Daí segue naturalmente o questinamento: como apresentá-lo ao aluno do ensino médio? Uma proposta seria, intuitivamente, apresentá-lo por meio de um exemplo particular mais simples. Considere a função quadrática $f(x)=x^{2}$ que tranforma a progressão aritmética

$$
1,2,3,4, \ldots, n, n+1, \ldots
$$

na sequência

$$
1,4,9,16, \ldots, n^{2},(n+1)^{2}, \ldots,
$$

que não é uma progressão aritmética. Entretanto, examinando-se as diferenças entre os termos consecutivos desta última sequência, encontra-se

$$
3,5,7, \ldots, 2 n+1, \ldots,
$$

que é uma progressão aritmética.

De forma geral, é possível verificar sem maiores dificuldades que essa característica constitui uma propriedade exclusiva das funções quadráticas (ver [2], por exemplo, para mais detalhes).

\section{Atividades}

Nas atividades seguintes, propõe-se a exploração dinâmica das variações funcionais - expressas por grandezas geométricas - como subsídio para a caracterização das funções quadráticas, antes mesmo de traçar seus gráficos, sem a mediação das leis de formação ou tabelas. Além disso, admite-se conhecido que uma função quadrática é contínua em todo o seu domínio.

Importante ressaltar que não é objetivo deste trabalho o aprofundamento nas instruções para as construções geométricas no GeoGebra. Para os leitores interessados na reprodução das atividades propostas, seguem no ANEXO os endereços eletrônicos para acesso/download as/das mesmas. Além disso, convém destacar que as Atividades 1 e 2 foram adaptadas dos concursos de seleção para o ingresso ao Programa de Pós-Graduação em Ensino de Matemática ${ }^{3}$ da Universidade Federal do Rio Grande do Sul, Exames 2012 (questão 2) e 2010 (questão 2), respectivamente, com objetivos diversos dos deste trabalho.

\footnotetext{
3 http://www.mat.ufrgs.br/ ppgem/
} 


\subsection{Atividade 1}

Considere um círculo $C_{1}$ cujo raio mede 2 unidades de comprimento. Seja $A P$ um diâmetro qualquer de $C_{1}$ (conforme Figura 1). Marque um ponto livre $O$ no segmento $A P$ tal que $O P$ seja o raio de um círculo $C_{2}$, tangente internamente ao círculo $C_{1}$ no ponto $P$. Como varia a área compreendida entre os dois círculos em função do raio $O P$ ?

Para explorar as ideias intuitivas da relação de dependência da área $A$, compreendida entre os dois círculos, em função da medida do raio $r=|O P|$ do círculo $C_{2}$, pode-se usar os recursos disponíveis do GeoGebra para construir os dois círculos - considerando satifeitas as condições dadas - e, numa planilha de cálculo do próprio GeoGebra (que tem funcionamento similar ao das planilhas eletrônicas mais usuais), registrar essa variação tomando-se um $\Delta r$ fixo (veja a Figura 1).

Buscando caracterizar uma função quadrática associada ao comportamento dessa variação, é importante observar que o valor de $\Delta r$ representa a razão de uma progressão aritmética. Sendo assim, em virtude do Teorema 1, deve-se verificar se a sequência numérica cujos termos representam os valores relativos às áreas obtidas em função das medidas dos respectivos raios, $r$ (coluna $\mathrm{B}$ da planilha), representa uma progressão aritmética de segunda ordem. Desta forma, aproveitando a mesma planilha de cálculos, registram-se, portanto, as diferenças $D[i] \mathrm{s}, D[i]=C[i+1]-C[i]$, dos termos consecutivos da sequência numérica estabelecida na coluna $\mathrm{C}$ da planilha, conforme ilustrado na Figura 1.

Convém destacar que as limitações técnicas do computador, em especial no que tange ao cálculo aproximado do número $\pi$, podem produzir resultados inesperados. Eventualmente, os $D[i]$ 's podem não ser exatamente iguais.

Note que para garantir que seja satisfeita a condição de tangência entre os círculos, a medida do raio $r$ do círculo $C_{2}$ deve variar entre 0 e 2. Recorrendo aos recursos computacionais do GeoGebra, é possível proceder uma alteração no valor de $\Delta r$ com o intuito de validar (ou não) a conjectura de que essa relação funcional caracteriza uma função quadrática. Para isso, clique em N (Figura 1) e movimente o cursor (direita ou esquerda) para, então, redefinir o valor de $\Delta r$.

Fixando-se um novo $\Delta r$, por exemplo 0.1, tem-se uma nova planilha na qual se pode observar que as diferenças $D[i]$ s também são constantes, indicando, portanto, que a sequência dos valores associados às areas, nessa nova situação, também constituem termos de uma progressão aritmética de segunda ordem. Observa-se que nessa situação o número racional -0.06283 é a melhor aproximação para os $D[i] \mathrm{s}$ (Figura 2).

Agora, observando a variação dos valores que constituem a relação de dependência funcional, explicitada nas planilhas, é possível conjecturar que a função transforma uma progressão aritmética ordinária não constante (de razão $\Delta r$ ) em uma progressão aritmética de segunda ordem não degenerada. Isso significaria que, segundo o teorema da caracterização das funções quadráticas, a função que a cada raio $r$ associa o valor da área $A$ correspondente é quadrática.

Cabe ressaltar, também, que os resultados apresentados pelo computador não podem servir como critério de verdade matemática, mas, sim, como subsídio para se estabelecer conjecturas sobre o modelo que se está estudando. Portanto, há uma necessidade da comprovação dos resultados embasada em argumentação formal.

Para verificar a validade dessa conjectura pode-se recorrer, então, à expressão algébrica associada à função quadrática. Para isso, sejam $r$ um raio qualquer escolhido no intervalo [0,2] e $A$ a área 


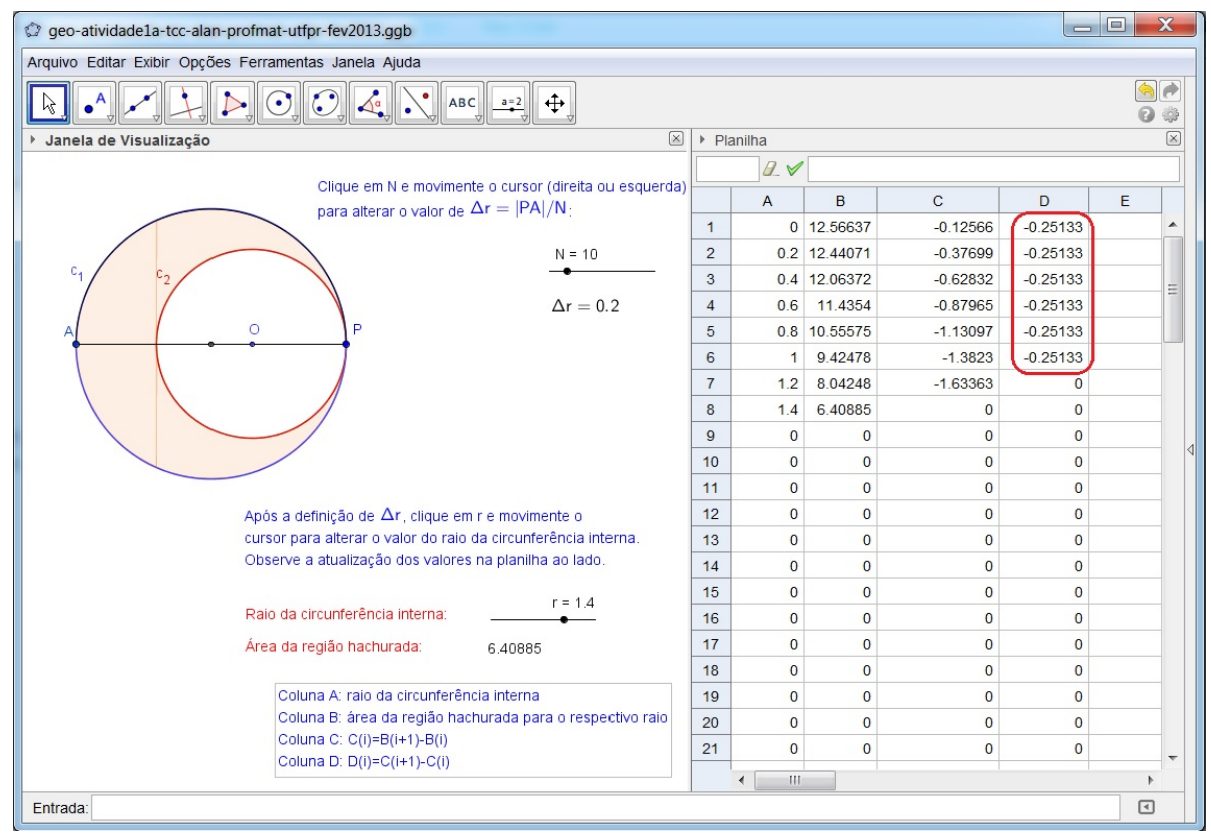

Figura 1: Ilustração da variação da área $A$ em função do raio de $C_{2} ; \Delta r=0.2$.

correspondente. Sabendo que a fórmula $\pi r^{2}$ define a área de um círculo cujo raio mede $r$ e que o círculo $C_{1}$ tem raio fixo medindo 2 unidades de comprimento, pode-se, sem dificuldade, deduzir a expressão da função área, $A$ :

$$
\begin{aligned}
A:[0,2] & \rightarrow \mathbb{R} \\
r & \mapsto \pi\left(4-r^{2}\right) .
\end{aligned}
$$

Portanto, segue que a função área $A$ é, realmente, quadrática.

As características intrínsecas do GeoGebra, no que diz respeito à sua condição de conector dinâmico de múltiplas representações, permitem observar a variação da função área graficamente, construindo-se o gráfico dessa função usando a opção lugar geométrico, possibilitando realizar um estudo mais geral dessa função (veja a Figura 3).

\subsection{Atividade 2}

Considere um quadrado $A B C D$ cuja diagonal mede 6 unidades de comprimento. Marque um ponto livre $H$ no segmento $A C$ e, em seguida, construa um segmento de reta paralelo à diagonal $B D$ que contenha o ponto $H$ e cujas extremidades estejam sobre os lados do quadrado. Seja $P$ o polígono convexo, inscrito ao quadrado (conforme Figura 4), cujos vértices são as extremidades desse segmento de reta e o vértice $A$ do quadrado. Considerando $h=|A H|$, como varia a área do polígono $P$ em função de $h$ ?

Seja $A$ a medida da área do polígono convexo $P$. Para explorar as ideias intuitivas da relação de 


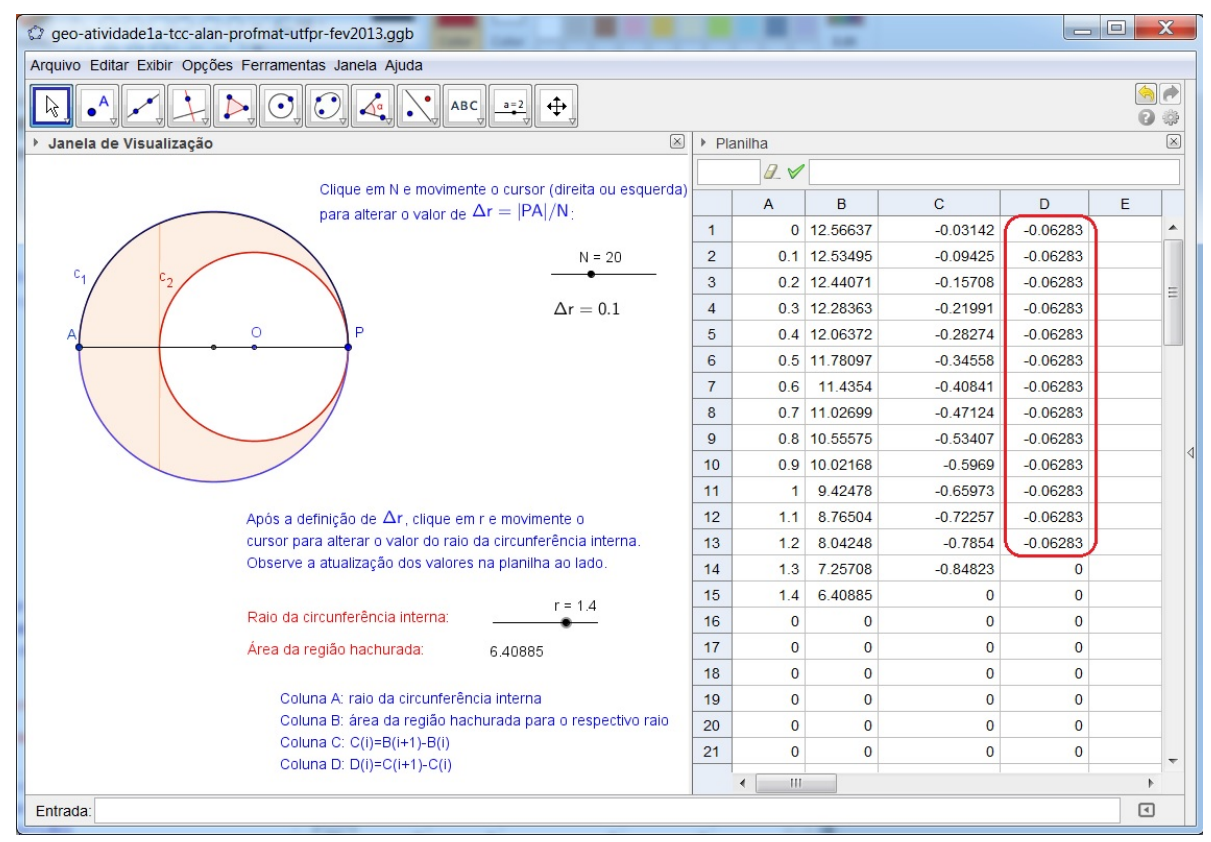

Figura 2: Ilustração da variação da área $A$ em função do raio de $C_{2} ; \Delta r=0.1$.

dependência da área $A$ em função da medida $h$, usando-se os recursos disponíveis do GeoGebra, deve-se, em primeiro lugar, construir o quadrado $A B C D$ e, em seguida, o polígono $P$ - considerando satisfeitas as condições dadas - e, na planilha de cálculo, registrar essa variação tomando-se um $\Delta h$ fixo qualquer (veja a Figura 4 ).

Da geometria do problema segue que para $h \leq 3$ o polígono $P$ assume o formato de um triângulo retângulo. Por outro lado, para $h \in] 3,6[$ o polígono $P$ assume o formato de um pentágono (Figura 5). É fato que se $h=6$, então o polígono $P$ coincide com o quadrado $A B C D$. Desta forma, é razoável afirmar que a função área, $A$, é definida por duas sentenças (funções de $h$ ). Deseja-se verificar se o estudo do comportamento da variação dessa relação funcional satisfaz as premissas do teorema de caracterização de funções quadráticas. Assim sendo, sejam $A_{1}$ a área do polígono $P$ quando o mesmo assume o formato de triângulo retângulo, e $A_{2}$ a área do polígono quando este assume o formato de um pentágono. Analisando os valores das funções, observa-se que ambas são monótonas crescentes em todo o seu domínio, pois tanto os valores de $A_{1}$ quanto os de $A_{2}$ aumentam quando $h$ aumenta. Também, admite-se intuitivamente a continuidade da função área. Além disso, analisando os valores das funções explicitados nas planilhas constantes nas Figuras 4-5, pode-se conjecturar que, para $\Delta h$ fixo, as funções definidas nesses intervalos transformam uma progressão aritmética ordinária de razão $\Delta h$ em uma progressão aritmética de segunda ordem não degenerada. Assim, é possível afirmar que a função área, $A(h)$, é definida por duas sentenças que são funções quadráticas.

Importante salientar que para cada $\Delta h$ fixado, nota-se que com o aumento dos valores de $h$ temse que $\Delta A_{1}$ (variação de $A_{1}$ ) cresce e $\Delta A_{2}$ decresce. Isso indica que a função $A_{1}$ cresce com concavidade para cima e a função $A_{2}$ cresce com concavidade para baixo. Pode-se verificar esse 


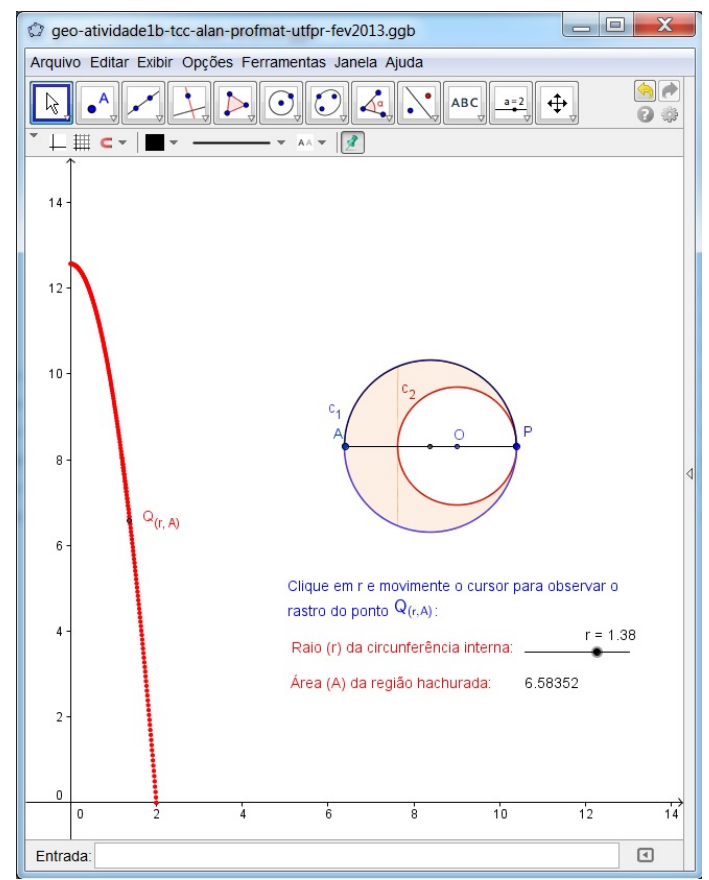

Figura 3: Ilustração do gráfico da área, $A$, em função do raio $r=|O P|$.

comportamento construindo-se o gráfico da função $A$ com o uso dos recursos do GeoGebra (veja Figura 6). Note que para $h=3$ tem-se um ponto de inflexão e, por conseguinte, há uma mudança na concavidade da função área $A$.

É fato que a representação algébrica também pode ser usada para estudar o comportamento da variação da área do polígono $P$ em função do valor de $h$. Com efeito, como a função área $A$ é definida por duas sentenças, pode-se proceder a análise em duas situações:

- Determinação da lei de formação da função $A_{1}$ - Observe que o triângulo retângulo em questão é isósceles, pois é semelhante ao triângulo $A B D$. Deste modo, considerando que $A_{\triangle}$ é a medida da área do triângulo de vértices $A B D$, segue, da semelhança, que:

$$
\frac{A_{1}(h)}{A_{\triangle}}=\left(\frac{2 h}{6}\right)^{2}, \quad \text { ou seja, } \quad A(h)=h^{2} .
$$

- Determinação da lei de formação da função $A_{2}$ - Note que a área do pentágono $A_{2}$ é igual à área do quadrado, subtraindo-se $A_{1}(6-h)$. Deste modo, segue que:

$$
\begin{aligned}
A_{2}(h) & =18-(6-h)^{2}= \\
& =\left(18-\left(36-12 h+h^{2}\right)\right)=-h^{2}+12 h-18 .
\end{aligned}
$$

Pode-se, então, concluir que a função $A:[0,6] \rightarrow \mathbb{R}$, que representa a área do polígono $P$ em 


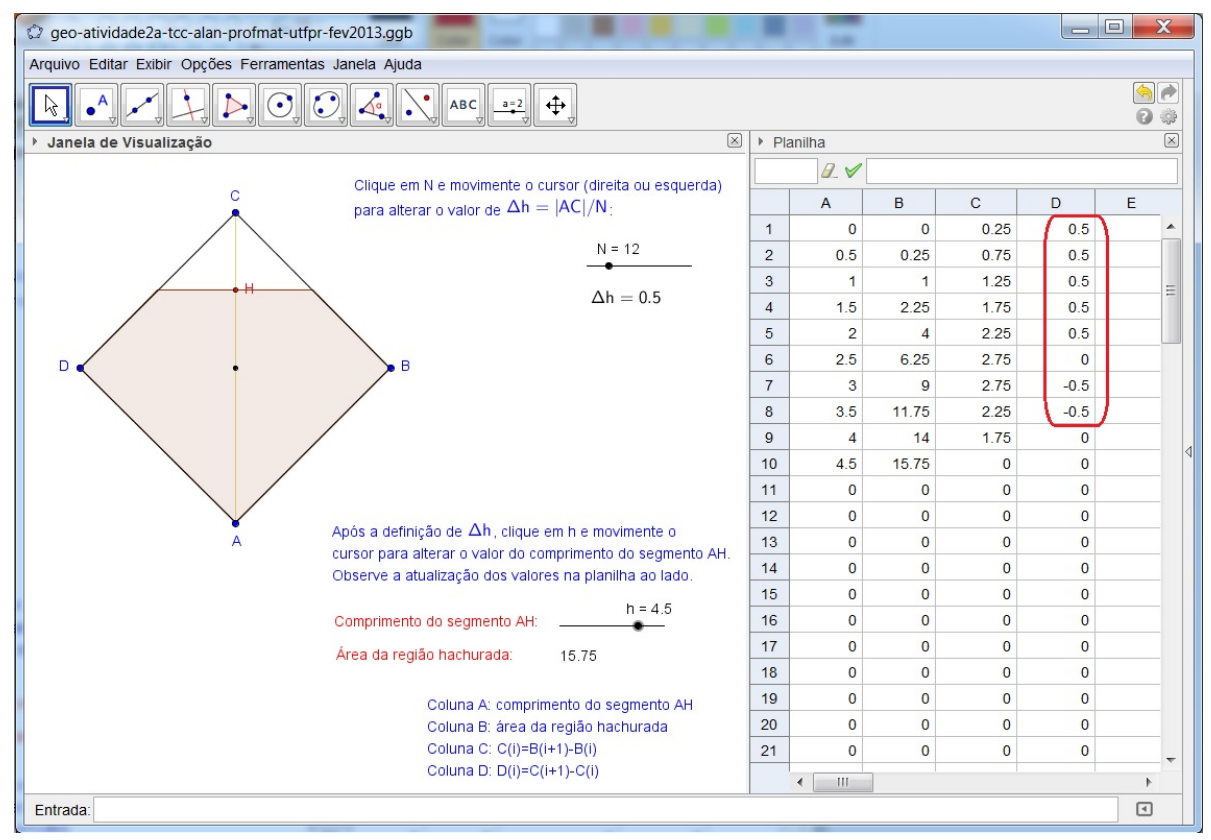

Figura 4: Ilustração da variação da área $A$ em função de $h=|A H| ; \Delta h=0.5$.

função do valor da medida $h$, é definida por

$$
A(h)=\left\{\begin{array}{rll}
h^{2}, & \text { se } & 0 \leq h \leq 3 \\
-h^{2}+12 h-18, & \text { se } & 3<h \leq 6,
\end{array}\right.
$$

uma função quadrática (por partes).

\subsection{Atividade 3}

Considere um setor circular de ângulo central reto e cujo raio $O A$ mede 5 unidades de comprimento. Marque um ponto livre $H$ no segmento $O A$ de modo que o segmento $O H$ seja a base de um retângulo inscrito no setor (conforme Figura 7). Como varia a área desse retângulo em função da medida da sua base?

Seja $A$ a medida da área do retângulo de base $O H$, inscrito no setor, e $h=|O H|$, a medida de sua base. De maneira análoga às Atividades 1 e 2 , é possível explorar as ideias intuitivas da relação de dependência da área $A$, em função da medida $h$, usando-se os recursos disponíveis do GeoGebra. Para isso, deve-se, em primeiro lugar, construir o setor circular e, em seguida, o retângulo de base $O H$. Em seguida, na planilha de cálculo registram-se os valores relativos a essa variação considerando-se um $\Delta h$ fixo qualquer (veja a Figura 7 ).

É interessante notar que os recursos computacionais podem, e devem, ser usados como subsídio para a argumentação matemática no sentido de constatar que a relação de dependência funcional tratada nesse modelo não caracteriza uma função quadrática. Sabe-se, pelo teorema de caracterização das 


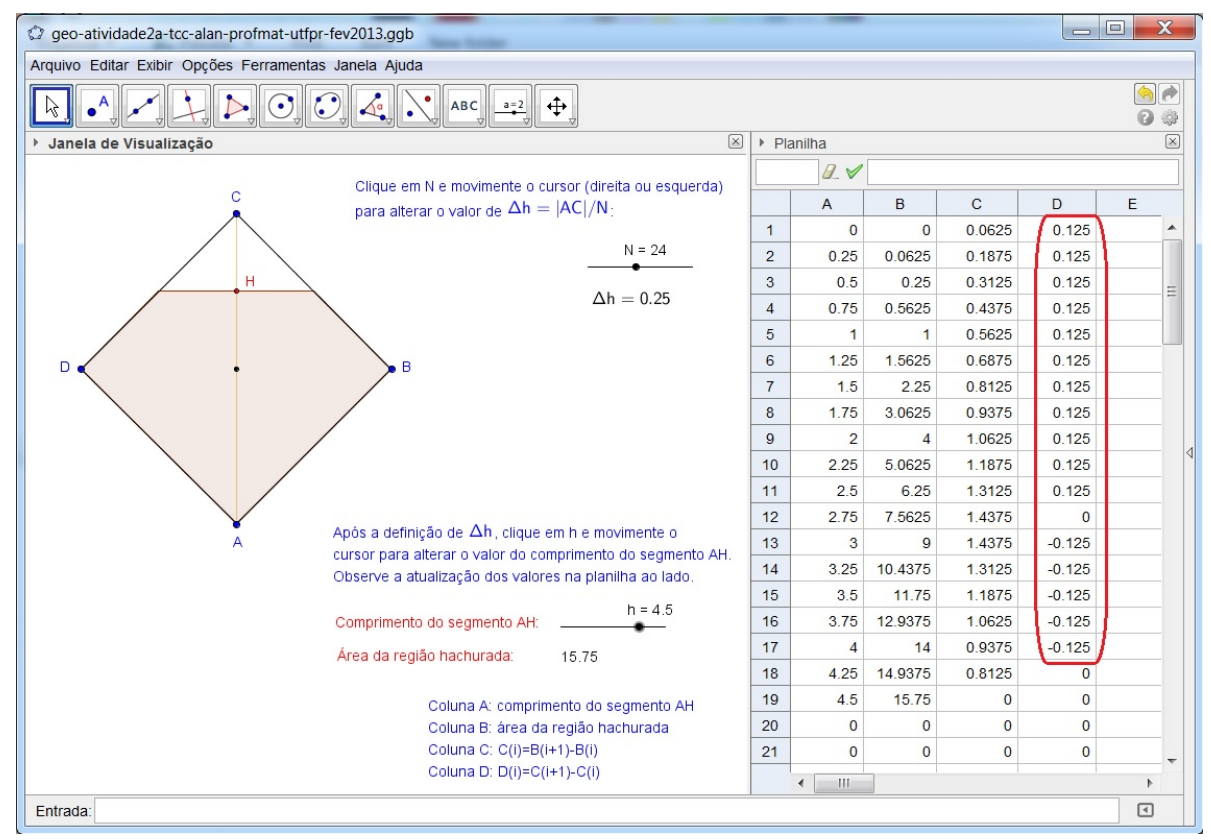

Figura 5: Ilustração da variação da área $A$ em função de $h=|A H| ; \Delta h=0.25$.

funções quadráticas, que a condição necessária para que uma função contínua seja quadrática é que toda progressão aritmética ordinária seja transformada pela função em uma progressão aritmética de segunda ordem não degenerada. De fato, analisando os valores da função explicitados na planilha, pode-se verificar que a função $A$ não é quadrática, pois a progressão aritmética de razão $\Delta h=0.25$ não foi transformada numa progressão aritmética de segunda ordem, uma vez que os $D[i]$ 's não são constantes, conforme observado na coluna $\mathrm{D}$ da planilha na Figura 7.

Além disso, no sentido de se estabelecer uma argumentação matemática mais fundamentada, podese ratificar a conclusão anteriormente estabelecida fazendo-se uso dos recursos computacionais do GeoGebra que oportunizam integrar os diversos registros do conhecimento. Destarte, construindo o gráfico dessa função como lugar geométrico, percebe-se que a curva descrita (Figura 8) não é simétrica, indicando, portanto, que o gráfico não é uma parábola. Desta forma, pode-se afirmar que a área do retângulo em função da medida da sua base, como já constatado anteriormente, não caracteriza uma função quadrática.

Construindo-se esse modelo em um sistema de coordenadas cartesianas, pode-se representar algebricamente a relação entre a área do retângulo em função da medida da sua base. Tome o vértice do setor circular coincidindo com a origem do sistema de coordenadas de tal forma que a base do retângulo esteja sobre o eixo das abscissas. Desta forma, segue que a equação do arco de circunferência $\overparen{A B}$ é dada por

$$
(\lambda): y^{2}+x^{2}=25, \quad 0 \leq x \leq 5 .
$$

Daí segue que para todo $P(x, y)$ em $(\lambda), y=\sqrt{25-x^{2}}, \quad 0 \leq x \leq 5$. 


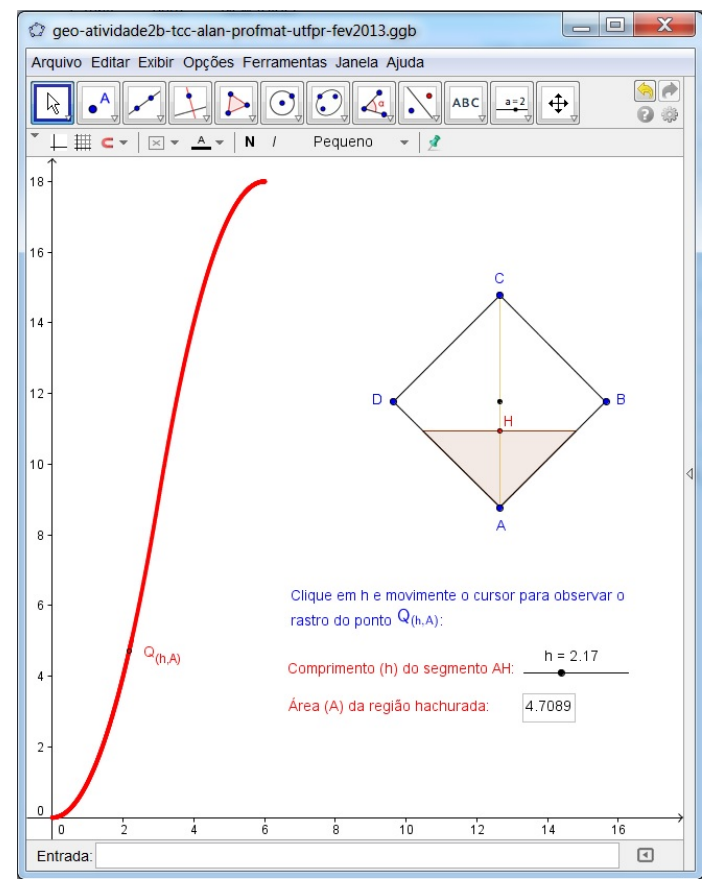

Figura 6: Ilustração do gráfico da área, $A$, em função de $h=|A H|$.

Assim sendo, pode-se observar que para cada valor $h$ da base tem-se um retângulo de altura $\sqrt{25-h^{2}}$ e, por conseguinte, a sua área é dada por:

$$
A(h)=h \sqrt{25-h^{2}} .
$$

\section{Considerações finais}

Indubitavelmente, o conceito de função quadrática é por si só bastante complexo para o aluno do ensino médio, pois envolve outros conceitos igualmente abstratos como domínio, contradomínio e imagem. Ao final dessas atividades, pode-se constatar a importância do uso do GeoGebra como recurso didático de apoio para se trabalhar a percepção intuitiva das funções reais, como no caso a função quadrática, antes de analisá-las através das representações algébricas e gráficas. Além disso, reunindo recursos de geometria dinâmica, álgebra e cálculo num mesmo programa, e com o mesmo grau de importância, o GeoGebra promove a integração dos diversos registros que permitem ao estudante ratificar as suas conjecturas.

Convém ressaltar que o conceito de progressão aritmética é um pré-requisito ao teorema da caracterização das funções quadráticas e, caso o professor opte também por utilizar as atividades propostas com seus alunos, ele deve introduzir anteriormente o conceito, mesmo que ainda de forma superficial, uma vez que tal conceito é simples e não seria empecilho para a aplicação das atividades. 


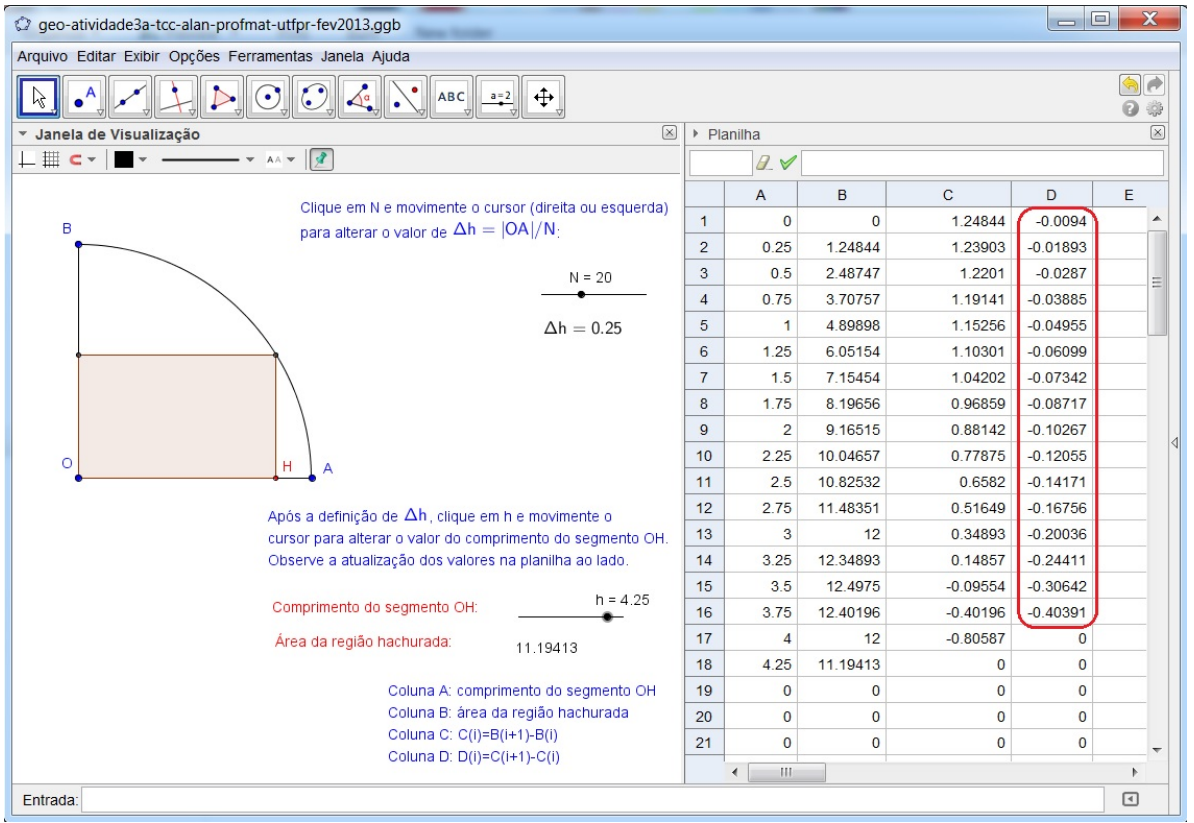

Figura 7: Ilustração da variação da área $A$ em função de $h=|O H| ; \Delta h=0.25$.

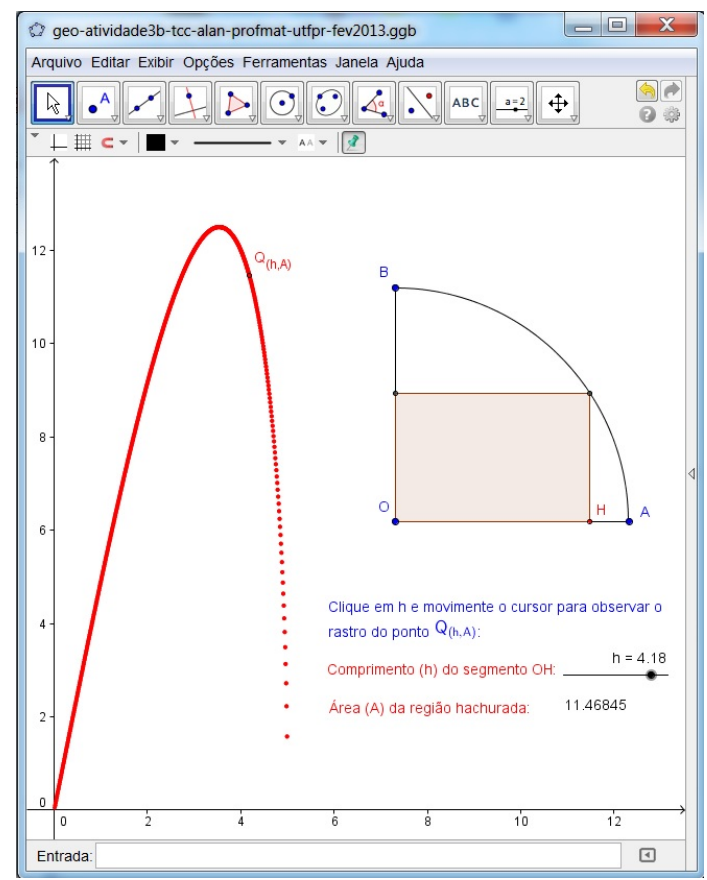

Figura 8: Ilustração do gráfico da área, $A$, em função de $h=|O H|$. 


\section{Referências}

[1] Giraldo, V. Integrando Geometria e Funções: Gráficos Dinâmicos. Revista do Professor de Matemática, v. 79, n. 3, p. 39-46, 2012.

[2] Lima, E. L. et al. A Matemática do Ensino Médio. Nona edição. Coleção do Professor de Matemática, CPM13, v. 1, Rio de Janeiro: Sociedade Brasileira de Matemática, 2006.

[3] Oliveira, A. G.; Dorini, F. A. Funções e Geometria: O Uso de Ambiente de Geometria Dinâmica como Subsídio para A Caracterização das Funções Quadráticas. Dissertação, Mestrado Profissional em Matemática em Rede Nacional - PROFMAT, Universidade Tecnológica Federal do Paraná, Curitiba, 2013.

\section{A. Anexo}

Segue o link para acesso (download) à coleção das Atividades 1, 2 e 3, elaboradas em GeoGebra, apresentadas no desenvolvimento do trabalho.

\section{http://www.geogebratube.org/collection/show/id/3056.}

Individualmente, as atividades podem ser acessadas em:

Atividade 1(a): http://www.geogebratube.org/material/show/id/31133;

Atividade 1(b): http://www.geogebratube.org/material/show/id/31132;

Atividade 2(a): http://www.geogebratube.org/material/show/id/31131;

Atividade 2(a): http://www.geogebratube.org/material/show/id/31130;

Atividade 3(a): http://www.geogebratube.org/material/show/id/31127;

Atividade 3(b): http://www.geogebratube.org/material/show/id/31082.

Alan de Oliveira

Colégio Militar de Curitiba <alannaval@yahoo.com.br>

Fabio Dorini

Universidade Tecnológica Federal do Paraná - Curitiba $<$ fabio.dorini@gmail.com> 